\title{
Planet-planet scattering in circumstellar gas disks
}

\author{
F. Marzari ${ }^{1}$, C. Baruteau ${ }^{2}$, and H. Scholl ${ }^{3}$ \\ 1 Dipartimento di Fisica, University of Padova, via Marzolo 8, 35131 Padova, Italy \\ e-mail: marzari@pd.infn.it \\ 2 Astronomy and Astrophysics Department, University of California, Santa Cruz, CA 95064, USA \\ e-mail: clement . baruteau@ucolick .org \\ ${ }^{3}$ Laboratoire Cassiopée, Université de Nice Sophia Antipolis, CNRS, Observatoire de la Côte d'Azur, BP 4229, \\ 06304 Nice Cedex, France \\ e-mail: Hans.Scholl@oca.eu
}

Received 1 April 2010 / Accepted 24 April 2010

\begin{abstract}
Context. Hydrodynamical simulations of two giant planets embedded in a gaseous disk have shown that in case of a smooth convergent migration they end up trapped into a mean motion resonance. These findings have led to the conviction that the onset of dynamical instability causing close encounters between the planets can occur only after the dissipation of the gas when the eccentricity damping is over.

Aims. We show that a system of three giant planets may undergo planet-planet scattering when the gaseous disk, with density values comparable to that of the minimum mass solar nebula, is still interacting with the planets.

Methods. The hydrodynamical code FARGO-2D-1D is used to model the evolution of the disk and planets, modified to properly handle close encounters between the massive bodies.

Results. Our simulations predict a variety of different outcomes of the scattering phase, which includes orbital exchange, planet merging and scattering of a planet in a hyperbolic orbit.

Conclusions. This implies that the final fate of a multiplanet system under the action of the disk torques is not necessarily a packed resonant configuration.
\end{abstract}

Key words. planets and satellites: formation - protoplanetary disks - planetary systems

\section{Introduction}

A multi-planet system made of Jupiter-sized bodies may reach completion when the planets are still under the influence of the disk. In this scenario the mutual gravitational perturbations compete with the gravitational torques exerted on the planets by the disk. These torques cause planet migration, which is a strong source of mobility to the system and possibly causes the planets to come closer to each other. The mutual approach is supposed to end when a pair of planets is captured in a mean motion resonance (e.g. the 5:3, 3:2, 3:1 or $2: 1$ ). The most extensively studied scenario is that of two jovian mass planets embedded in a viscously evolving disk. Kley et al. (2004, 2005), Masset \& Snellgrove (2001) and Pierens \& Nelson (2008) have shown via self-consistent disk-planet hydrodynamical simulations that the two planets become trapped into a resonance and create a cavity encircling their orbits. This resonant configuration appears stable in these simulations, which argues in favor of a scenario where the resonance locking should be broken only after gas dissipation possibly by the end of the gas damping or by planetesimal scattering.

Damped $N$-body models have also been used to study the evolution of two planet systems where the disk-planet interaction is modeled as an external dissipative force. Lee \& Peale (2002), Beauge et al. (2006) and Lee et al. (2009) have shown that the resonance trapping is permanent if a strong damping of eccentricity is adopted. Even turbulence appears not to prevent the resonance capture (Rein \& Papaloizou 2009) in this scenario. The escape from resonance can occur only if the eccentricity damping term used in the model is relatively low and, after the resonance breaking, planets may have close encounters. This second approach characterizes the outcome of the simulations by Moorhead \& Adams (2005), where two planets may undergo planet-planet scattering after the escape from resonance trapping. However, hydrodynamical simulations have so far never shown such a behavior with the planets assumed to remain in resonance until the dissipation of the gas. The gas damping appears to be dominating over the mutual gravitational perturbations even for Jupiter size planets, which supports the idea that the preferred outcome of the evolution of a multiplanet system under the tidal action of the disk is a packed resonant system.

A system of three giant planets has proved to be dynamically more prone to instability and to develop crossing orbits (Weidenschilling \& Marzari 1996; Chatterjee et al. 2008) compared to the case of two planets. The mutual gravitational perturbations are stronger and may more easily overcome the disk-damping effect. The observed systems Ups And, $55 \mathrm{Cnc}$, HIP 14810, HD 74156, HD 160691 and HD 37124 also show that it is indeed possible to form more than two giant planets around solar type stars. For this reason we selected a scenario where three giant planets with masses close to that of Jupiter form in the disk and evolve under planet-disk and planet-planet interactions. We will show that the chaotic evolution, which is characterized by mutual close encounters between (giant) 

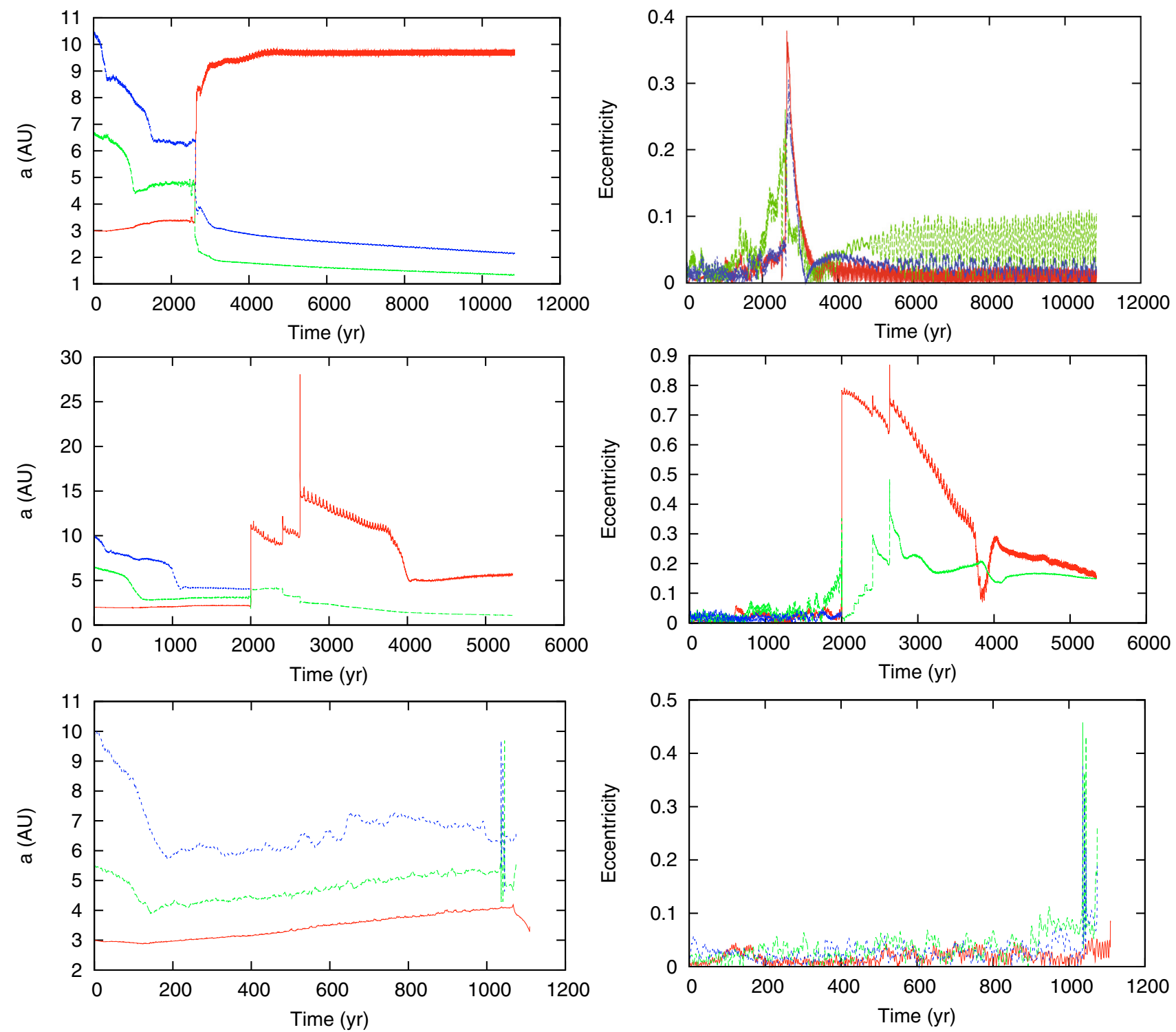

Fig. 1. Orbital evolution of the three planets while embedded in the disk. The top plots show a model where $\Sigma_{0}=0.4 \Sigma_{\mathrm{MMSN}}$ and $a_{1}=3.0 \mathrm{AU}, a_{2}=$ $6.7 \mathrm{AU}, a_{3}=10.5 \mathrm{AU}$. The middle plots show a case with $\Sigma_{0}=0.65 \Sigma_{\mathrm{MMSN}}$ and $a_{1}=2.0 \mathrm{AU}, a_{2}=6.5 \mathrm{AU}, a_{3}=10.0$ AU. The bottom plots illustrate the evolution when $\Sigma_{0}=\Sigma_{\mathrm{MMSN}}$ and $a_{1}=3.0 \mathrm{AU}, a_{2}=5.5 \mathrm{AU}, a_{3}=10.0 \mathrm{AU}$.

planets, is favored by planet migration. Our hydrodynamical simulations show that most of the time the planets are quickly captured in a three-body resonance during their migration, despite their rapid drift rate, but this may become unstable. The growth in eccentricity leads the planets into crossing orbits, and they begin to evolve under the effects of mutual approaches. The following chaotic phase ends up either with the ejection of a planet, planet-merging or orbital reconfiguration.

\section{Numerical set-up}

The evolution of the planets and disk was modeled with the hydrodynamical code FARGO-2D-1D (Crida et al. 2007) where the numerical integrator computing the planet orbits was updated with a variable stepsize so that mutual close encounters between the planets could be properly modeled. The 2D grid, which resolves the local disk-planet interactions, is surrounded by a $1 \mathrm{D}$ (non azimuthally resolved) grid that allowed us to simulate the disk's global evolution (Crida et al. 2007). The 2D grid used in the simulations to represent the disk has a logarithmic radial spacing extending from $r=0.5$ to $r=15$ AU. It is characterized by $N_{\mathrm{r}}=512$ elementary rings, and $N_{\mathrm{s}}=256$ sectors. The 1D grid spans a radial distance from $15 \mathrm{AU}$ outwards till $50 \mathrm{AU}$. The disk-aspect ratio $(\mathrm{H} / \mathrm{r})$ was set uniformly to $5 \%$ and constant in time because the disk was assumed to be locally isothermal. The gas kinematic viscosity is $v=10^{-5}$ in code-normalized units, which corresponds to an alpha parameter of $4 \times 10^{-3}$. The gas density profile declines as $r^{-1 / 2}$ and the value $\Sigma_{0}$ at $1 \mathrm{AU}$ was varied in the simulations, ranging from 0.25 to $1 \Sigma_{\text {MMSN }}$, the density at $1 \mathrm{AU}$ predicted for the minimum mass solar nebula. In the simulations presented here the three planets have masses equal to $1.5,0.9$ and 1.2 Jupiter masses, while the initial semimajor axes are selected to be between 2 and 10 AU. They are all started on circular orbits and do not accrete mass from the disk.

\section{Results}

Figure 1 illustrates the different behaviors we observed in our simulations. After an initial fast migration, the three giant planets become locked into a three-body mean motion resonance. 


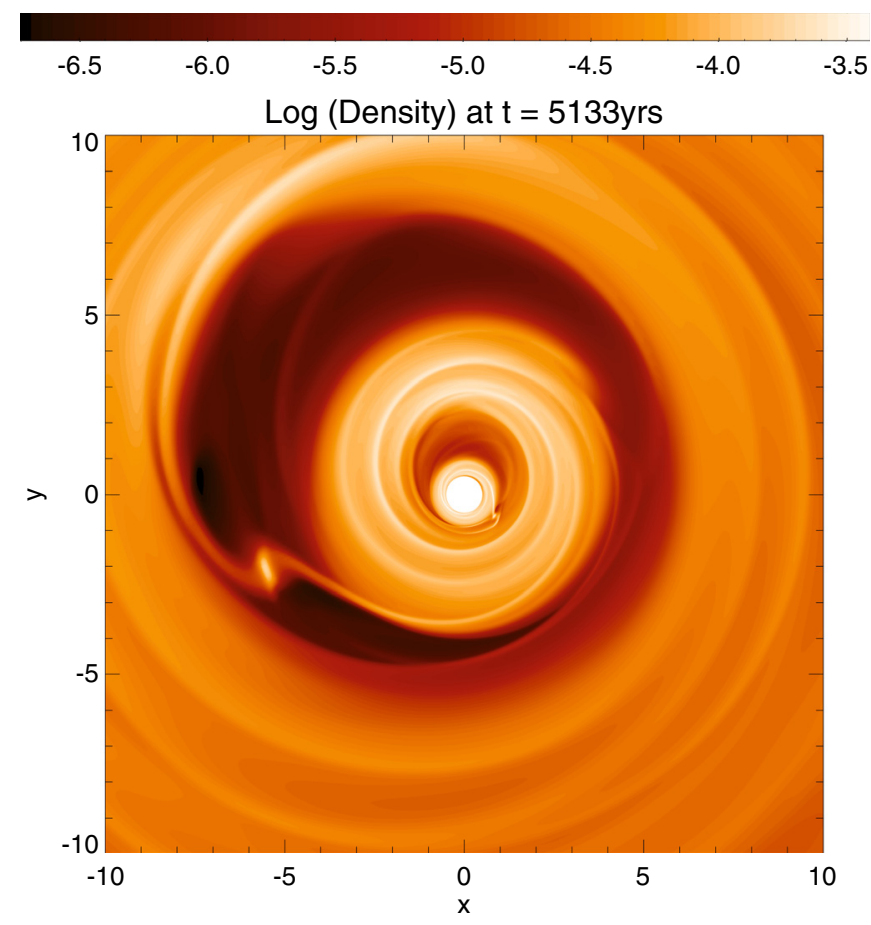

Fig. 2. Disk surface density distribution after the planet-planet scattering phase shown in Fig. 1 middle plots. The two planets have developed eccentric gaps.

However, the resonant trapping is not stable and the growth in eccentricity leads to planet-crossing orbits, which in turn start the chaotic phase. In the top plots we show the semi-major axis and eccentricity evolution of the three planets initially set on orbits with $a_{1}=3.0 \mathrm{AU}, a_{2}=6.7 \mathrm{AU}, a_{3}=10.5 \mathrm{AU}$ with $\Sigma_{0}=0.4 \Sigma_{\text {MMSN }}$. After repeated mutual encounters, an orbital exchange occurs in which innermost planet becomes the outermost one. In the middle plots, a case of planet merging is displayed, where the two surviving planets are left on mildly eccentric orbits. In this model the initial disk density is $\Sigma_{0}=$ $0.65 \Sigma_{\mathrm{MMSN}}$ and the planets are started at $a_{1}=2.0 \mathrm{AU}, a_{2}=$ 6.5 $\mathrm{AU}, a_{3}=10.0 \mathrm{AU}$. Very large eccentricities are achieved during the chaotic phase, but they are partly damped by the disk after the merging event. It is interesting to note that in this second case, after the planet-planet scattering, the two planets left on eccentric orbits are surrounded with eccentric gaps. This is illustrated in Fig. 2. The bottom plots refer to a simulation where two planets merge and are ejected out of the system on a hyperbolic orbit ( $\left.\Sigma_{0}=\Sigma_{\mathrm{MMSN}}, a_{1}=3.0 \mathrm{AU}, a_{2}=5.5 \mathrm{AU}, a_{3}=10.0 \mathrm{AU}\right)$.

Different set ups were tested to check the robustness of our findings. In one series of simulations, the mass of each planet grows from 0 to the final value on a timescale ranging from 10 to $1000 \mathrm{yrs}$, the latter being comparable with the rapid gas-infall timescale typical of the final stage of a giant planet growth. In another series, planets are first held on fixed circular orbits until they form gaps, after what they are let to evolve under diskplanet and planet-planet interactions. Very similar behaviors are observed in all these cases. The mutual gravitational interactions within a system of three planets force some eccentricity, which is not fully damped by the disk. In addition, the wakes excited by each planet tamper with the gap-cleaning process of the other planets. The two outermost planets in our simulations do not undergo slow type II migration. They experience strong torques from their co-orbital region which is not fully cleared. The fast migration (runaway migration, Masset \& Papaloizou 2003) triggered by corotation torques leads the planets close enough to be trapped into mean-motion resonance. But this is not stable and planet-planet scattering follows.

\section{Discussion and conclusions}

Although there two-planet systems in simple mean-motion resonances are known, there are also many systems that are either multiple and non-resonant, or that display evidence for scattering that would be suppressed if resonant capture was a ubiquitous process. We show that resonant capture is more easily evaded in a three-planet as opposed to a two-planet system, leading to a phase of planet-planet scattering. A major implication of planet-planet scattering in the gas disk is the significant rearrangement of planet orbits and eccentricity excitation, which may affect the subsequent evolution of the planets. As a consequence, the final mass distribution of planets as a function of the semi-major axis cannot be representative of the formation process. In addition, a closely packed planetary system where the multiple planets are all locked in mean-motion resonances is not the preferred outcome of planet migration, as previously argued. The chaotic phase may lead to different orbital configurations where the planets can be well separated into semi-major axis and stable even after the gas dissipation. Lower eccentricities are expected as the final state of the planet evolution because damping can occur after the dynamically active phase (Moeckel et al. 2008). However, it may not be the case for corotation resonance saturation. According to Goldreich \& Sari (2003); Ogilvie \& Lubow (2003) a planet may experience a net growth of eccentricity via disk-planet interaction if its eccentricity is not small to begin with. This would be the case for a planet at the end of the chaotic phase.

Additional simulations are done to perform a deeper exploration of the parameter space to identify the initial configurations of the planets and disk that lead to planet-planet scattering rather than to a stable resonant trapping.

Acknowledgements. We thank Phil Armitage for his useful comments and suggestions as referee of the paper.

\section{References}

Beaugé, C., Michtchenko, T. A., \& Ferraz-Mello, S. 2006, A\&A, 365, 1160 Chatterjee, S., Ford, E. B., Matsumura, S., \& Rasio, F. A. 2008, ApJ, 686, 580 Crida, A., Morbidelli, A., \& Masset, F. 2007, A\&A, 461, 1173 Goldreich, P., \& Sari, R. 2003, ApJ, 585, 1024

Kley, W., Peitz, J., \& Bryden, G. A. 2004, A\&A, 414, 735

Kley, W., Lee, M. H., Murray, N., \& Peale, S. J. 2005, A\&A, 437, 727

Lee, A. T., \& Peale, S. 2002, ApJ, 567, 596

Lee, A. T., Thommes, E. W., \& Rasio, F. A. 2009, ApJ, 691, 1684

Masset, F., \& Papaloizou, J. C. B. 2003, ApJ, 588, 494

Masset, F., \& Snellgrove, M. 2001, MNRAS, 320, L55

Moeckel, N., Raymond, S. N., \& Armitage, P. 2008, ApJ, 688, 1361

Moorhead, A. V., \& Adams, F. C. 2005, Icarus, 178, 517

Pierens, A., \& Nelson, R. P. 2008, A\&A, 482, 333

Ogilvie, G. I., \& Lubow, S. H. 2003, ApJ, 587, 398

Rein, H., \& Papaloizou, J. C. B. 2009, A\&A, 497, 595

Weidenschilling, S. J., \& Marzari, F. 1996, Nature, 384, 619 